\title{
Neglected posterior hip dislocation: management of an 18 years old female
}

\author{
Sonowal Kiran ${ }^{1}$, Singha Sodagor ${ }^{2}$, Buragohain CR $^{3}$
}

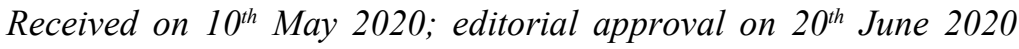

\begin{abstract}
Neglected traumatic dislocations of the hip are one of the disabling condition in lower extremity which is seldom found in adults. Also, it is not an uncommon condition seen in developing countries. Its treatment is often difficult and controversial. Here we present 18 years old female who presented to our hospital with 7 years old posterior hip dislocation sustained following fall from a bicycle. She had gone through multiple treatments for her dislocated hip without any success. The patient was managed by Girdlestone osteotomy and skeletal traction followed by total hip replacement (THR) with uncemented acetabular cup and cemented femoral stem. Her Harris hip score improved from 48 (preoperative) to 87 (postoperative). On follow-up, she had a good range of movement of the hip with no pain, deformity and limb length discrepancy. Girdlestone osteotomy and skeletal traction followed by total hip replacement (THR) provide promising results in neglected hip dislocation.
\end{abstract}

Keywords: Girdlestone Osteotomy; Total Hip Replacement; Harris Hip Score.

\section{INTRODUCTION}

Neglected traumatic dislocations of the hip are rare in adults. However, in developing countries, unreduced traumatic dislocations are not uncommon. They are usually the result of a motor vehicle accident, often combined with multiple trauma including head injury, fracture of the ipsilateral femur or bilateral injuries, which may detract attention from the dislocation. ${ }^{1}$ Neglected hip dislocations occur in situations when the patient does not or cannot seek adequate medical care. As such, chronic dislocations may be observed in patients with high pain tolerance, patients with the decreased cognitive ability to recognize or verbalize their pain and patients with additional injuries that are more obvious or life-threatening. ${ }^{2}$ In developing countries, patients usually attend hospital many days after trauma, having often received alternate therapy before. Treatment of neglected dislocation of the hip becomes more difficult to manage as time progresses because of the soft tissues contractures, adhesions, fibrofatty tissue filling of the acetabulum, and presence of myositis ossificans. ${ }^{3}$ Reduction of neglected dislocation of the hip is not only difficult but results in avascular necrosis and arthritis. Total hip replacement (THR) is recommended for hip dislocations with a duration of more than 3 months. ${ }^{2}$

A complete understanding of the factors that play a role in the aetiology of the instability of hip and a clear knowledge of treatment options are mandatory for the surgeon tackling this injury. Girdlestone osteotomy and skeletal traction followed by total hip replacement (THR) is a viable option for neglected dislocation hip.

\section{CASE REPORT}

18 years old female sustained a fall from a bicycle, resulting in an injury of her left hip, causing pain and an inability to weight bear. She saw a local doctor, who diagnosed a dislocation of her hip and attempted a reduction, which failed, as the hip remained painful and unstable. Six-months after the injury, the patient consulted an orthopaedic surgeon, who
Address for Correspondence:
${ }^{1}$ Assistant Professor (Corresponding author)
Mobile: +919678906577
Email: kieronsonowal@gmail.com
${ }^{2}$ Post Graduate Trainee, ${ }^{3}$ Assistant Professor
Department of Orthopaedics
Jorhat Medical College and Hospital, Jorhat, Assam, India 
advised to undergo an open reduction of the hip, but patient abandoned treatment.

\section{MANAGEMENT}

We saw this patient 7 years after the initial injury complaining of hip pain. She was walking with an antalgic gait and had a decreased range of motion of her hip. The limb was in fixed adduction and internal rotation. She had a leg length discrepancy due to $5 \mathrm{~cm}$ shortening of the left lower extremity. Her hip movements were restricted and painful. Radiographs confirmed a persistent dislocation of the hip, with a false acetabulum in the left supra-acetabular region as shown in Figure 1.

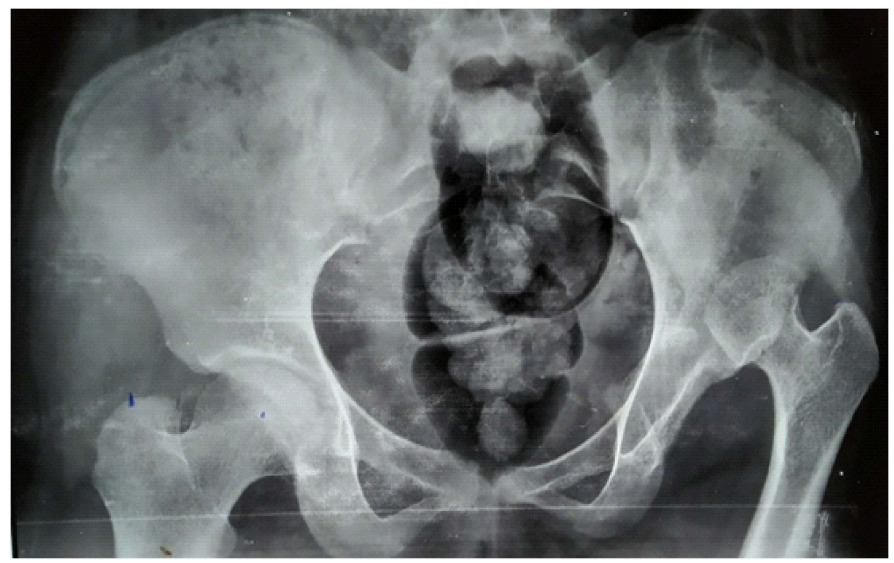

Figure 1 Pre-operative x-ray of the pelvis with Both Hip showing neglected left posterior hip dislocation

Initially, open reduction was planned for this patient with 2 weeks before skeletal traction. During surgery, we encountered soft tissues contractures, adhesions, fibrofatty tissue filling of the acetabulum, and irregular sclerotic cortex with enlargement of the femoral head. This anatomical distortion made it almost impossible open reduction of hip. We decided to clear the acetabulum of fibrous tissues and did Girdlestone osteotomy and soft tissue release (Figure 2). Postoperative skeletal traction with increasing weight was

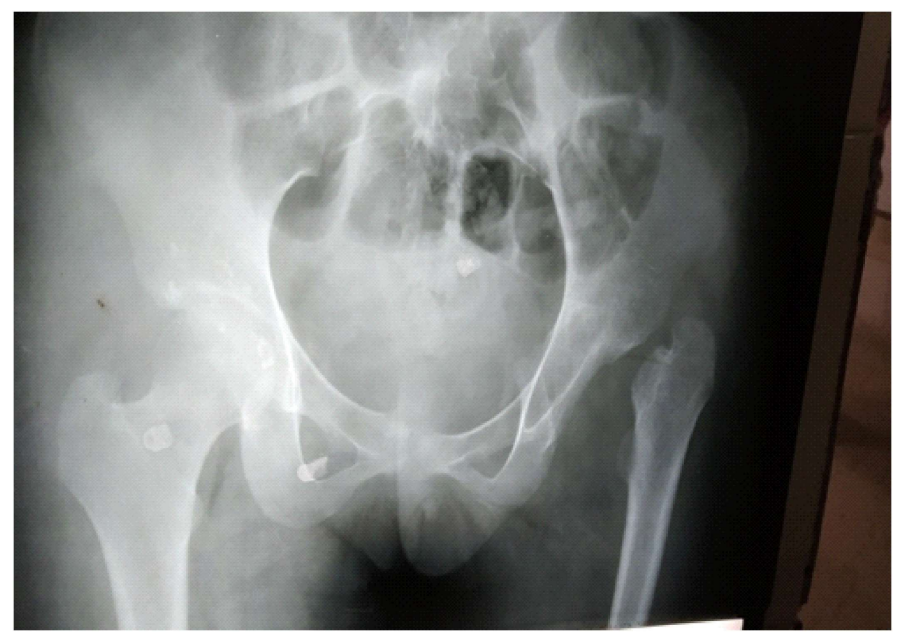

Figure 2 X-ray of the pelvis with both hip after the first operation, showing post-Girdlestone osteotomy and skeletal traction given for about 2 months and serial weekly $\mathrm{X}$ rays were done. Traction was ceased when the greater trochanter was drawn at the level of the articular surface of the acetabulum (Figure 3). Then THR was done with uncemented acetabular cup and cemented femoral stem with prophylactic proximal femoral encircle (Figure 4). The leg lengths were equalized, and the postoperative recovery was uneventful. Roentgenograms showed satisfactory position and fitting of the prosthesis.

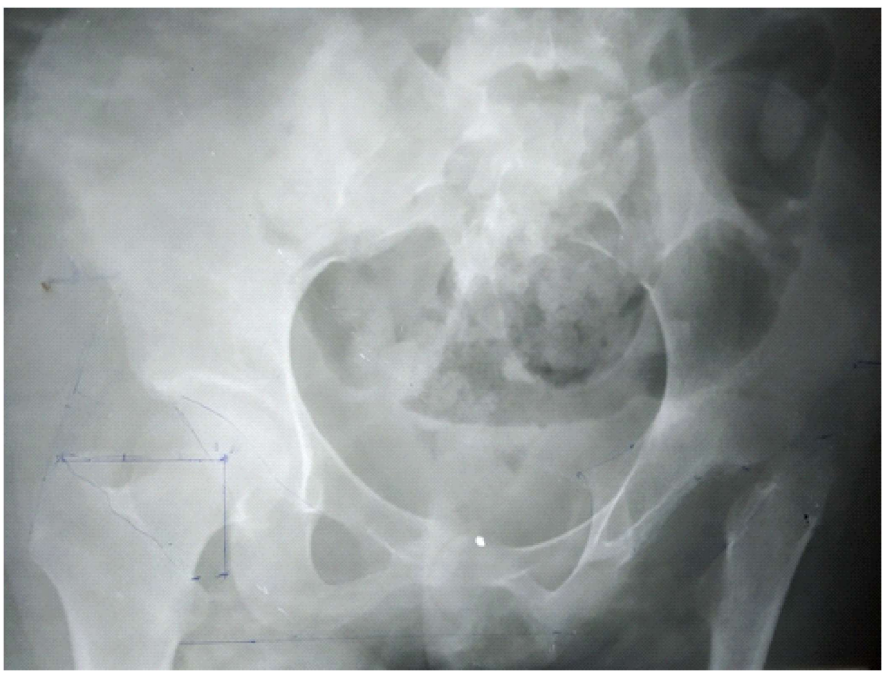

Figure $3 \mathrm{X}$-ray of the pelvis both hip after the greater trochanter was drawn at the level of the articular surface of the acetabulum

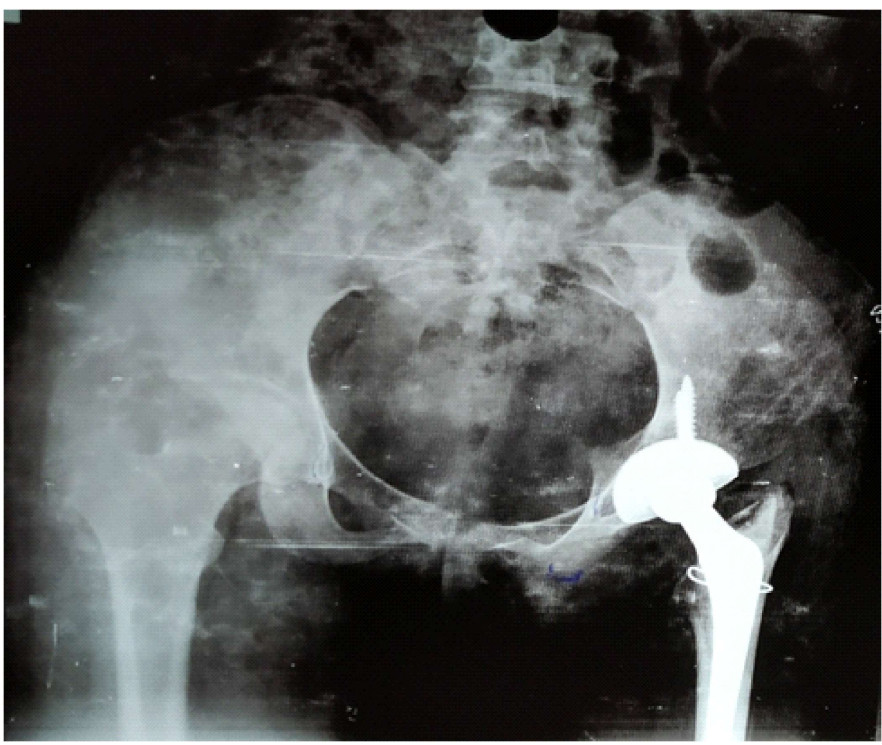

Figure 4 Post-operative pelvic both hip X-Ray of the patient after total hip replacement

Her physiotherapy was started soon postoperatively, and she was discharged on the fourteenth postoperative day. Her functional status improved on every successive follow-up visit, and the HHS score improved from 48 (preoperatively) to 87 (postoperatively). She had no pain, a mild residual Trendelenburg gait due to her abductor insufficiency and excellent function. 


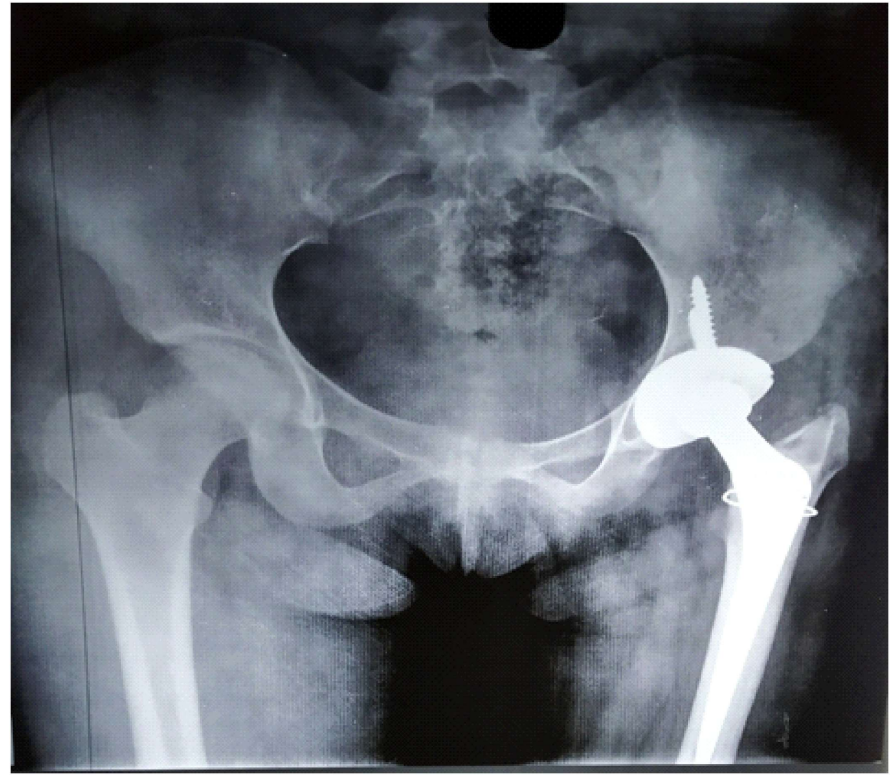

Figure 5 Three months Postoperative follow up pelvic X-Ray of the patient after total hip replacement

\section{DISCUSSION}

The ongoing discussion of the best treatment option for the management of neglected dislocation hip continues and remains controversial. Various studies reported different methods for operative treatment of neglected fracturedislocations including the use of a sub-trochanteric osteotomy, the Girdlestone procedure, hip arthrodesis, hemiarthroplasty and total hip replacement. ${ }^{4}$ All these procedures have their merits and give different outcomes. The results can be further altered by avascular necrosis of the femoral head which occurs in more than $50 \%$ of these cases. Garrett et al. ${ }^{2}$ have recommended total hip arthroplasty for hips with posterior dislocations classified as type IV (fracture of the acetabular rim and floor) or type $\mathrm{V}$ (fracture of the femoral head with or without other fractures) with dislocations for more than 3 months.

Pai recommended reduction under anaesthesia for patients where a dislocation had occurred three days to three weeks previously. 5

Banskota et al., ${ }^{4}$ in their study of eight cases with neglected posterior dislocation on hip reported good results in three cases treating the hip with an open reduction. They achieved a mean Harris hip score of 89 (range 84-96). Leg lengths were within $2 \mathrm{~cm}$ in seven of the eight cases, and one patient had a discrepancy greater than $2 \mathrm{~cm}$.

Verma et al., ${ }^{6}$ in his study of 14 cases neglected dislocation ranging from 14 days to 1 -year duration performed open reduction and reported excellent results in four cases, but also had poor outcomes in five cases.

In a study conducted by Berend et al., ${ }^{7}$ using a constrained THR, ten per cent were primary hip replacements in patients with an abductor dysfunction secondary to trauma or neuromuscular disease. They reported promising results, which would indicate that constrained THR to be a viable alternate option with abductor dysfunction with a high dislocation risk.

In a study conducted by Paavilainen et al., 100 cementless total hip replacements in 52 severely dysplastic and 48 dislocated hips, with some new technical solutions to the problems involved. In hips with tight flexor and abductor muscles or with deformities of the proximal femur, various osteotomies were performed. Final results were the generally good limited correction of limb length discrepancy may be achieved with THR. ${ }^{8}$

Patel et al., presented a case of 30 years-old male patients with neglected hip dislocation. The patient complained pain in the left hip for 2 years with fixed deformity of the hip, with a history of fall from a tree 15 years ago. The radiographs showed an obturator dislocation of the hip with obvious pseudo-acetabulum around the dislocated femoral head. Uncemented total hip arthroplasty was performed and after 2 years follow up, the patient was able to walk unaided without pain. ${ }^{9}$

Ilyas et al., treated 15 patients with chronic unreduced hip fracture-dislocations result from motor vehicle injury at King Faisal Specialist Hospital and Research Center. All underwent 1-stage total hip arthroplasty with bone grafting and then monitored for 36-96 months range. Although there were 2 dislocations, 1 transient peroneal nerve palsy, and 1 superficial infection, generally all patients had significantly decreased pain, increased function, and increased range-of-motion scores using the Merle d'Aubigné scoring system. ${ }^{\mathbf{1 0}}$

Kumar et al., reported a 31 years old female patient with neglected hip dislocation. The initial injury was 2 years before the patient's presentation to the hospital and had gone through many multiple treatments for her disease without any improvement. The patient was treated by constrained total hip replacement, resulting in improvement of Harris Hip Score from 48 to 81 . On follow up, the patient showed improved range of motion of hip joint without pain. ${ }^{11}$

\section{CONCLUSION}

Our case of 7 years old neglected dislocation of the hip treated by Girdlestone osteotomy and skeletal traction followed by total hip replacement (THR) with uncemented acetabular cup and cemented femoral stem total hip replacement which improves the patient's functional status successfully. Despite gaining 5-6 cm of limb length, the sciatic nerve was unaffected which is indirectly favouring Eggli et al., ${ }^{12}$ who found no correlation between the amount of lengthening and sciatic nerve palsy in a large study on THR.

We have not found any other case report with a 7-year-old neglected dislocated hip with contractures of the stabilizing muscles and pseudoarthrosis, as in our case, having achieved a satisfactory conclusion.

Funding: None declared.

Conflict of interest: None declared. 
Informed consent: Obtained.

\section{REFERENCES}

1. Hougaard K, Thomsen PB. Traumatic posterior fracture-dislocation of the hip with fracture of the femoral head or neck, or both. J Bone Joint Surg Am 1988;70:233-239. doi: 10.2106/00004623198870020-00011.

2. Garrett JC, Epstein HC, Harris WH, Harvey JP, Jr, Nickel VL. Treatment of unreduced traumatic posterior dislocations of the hip. J Bone Joint Surg Am 1979;61:2-6. doi: 10.2106/00004623-19796101000002.

3. Pugalenthi PV, Ramanathan S, Thanappan N, Sagar MV. A rare case of neglected unreduced posterior dislocation of hip managed with total hip arthroplastya case report. J Evolut Med Dent Scie 2017 Mar 20;6(23):1953-7.

4. Banskota AK, Spiegel DA, Shrestha S, Shrestha OP. Rajbhandary T-open reduction for neglected traumatic hip dislocation in children and adolescents. J Pediatr Orthop 2007;;27(2):187-191. doi:10.1097/ BPO.0b013e31802c547e.

5. Pai VS, Kumar B. Management of unreduced traumatic posterior dislocation of the hip: heavy traction and abduction method. Injury 1990;21:225-227. doi: 10.1016/0020-1383(90)90008-I.
6. Verma BP. Management of old unreduced traumatic dislocation of hip. Indian J Orthop 1975;9(2):69-80.

7. Berend KR, Lombardi AV, Jr, Mallory TH, Adams JB, Russell JH, Groseth KL. The longterm outcome of 755 consecutive constrained acetabular components in total hip arthroplasty examining the successes and failures. J Arthroplasty 2005;20(7 Suppl 3):93-102. doi: 10.1016/j.arth.2005.06.001.

8. Paavilainen T, Hoikka V, Salonen KA. Cementless total replacement for severely dysplastic or dislocated hips. J Bone Joint Surg 1990;72(2):205-11.

9. Patel S, Thimmegowda RB, John R, Kumar TS, Arjun RH. Total hip arthroplasty in neglected, obturator type, hip dislocation in a young adult-an unusual case and literature review. J Arthrosc Joint Surg 2017 Jan 1;4(1):50-3.

10. Ilyas I, Rabbani SA. Total hip arthroplasty in chronic unreduced hip fracture-dislocation. J Arthropl 2009 Sep 1;24(6):903-8.

11. Kumar S, Dahuja A, Narula MS, Garg S, Kaur R. Neglected hip dislocation: an unusual presentation, its management and review of the literature. Strat Trauma Limb Reconst 2017 Nov 1;12(3):189-92.

12. Eggli S, Hankemayer S, Muller ME. Sciatic nerve palsy after limb lengthening in THR. J Bone Joint Surg Br 1999;81(5):843-845. doi: 10.1302/0301620X.81B5.9610. 